\title{
Investigation of optically generated kink effect in GaAs-based heterojunction phototransistors
}

\author{
H.A. Khan ${ }^{1, a}$ and A.A. Rezazadeh ${ }^{2}$ \\ 1 Department of Electrical Engineering, Lahore University of Management Sciences, Lahore Cantt, 54792, Pakistan \\ 2 Microwave and Communication Systems Research Group, School of Electrical and Electronic Engineering, \\ The University of Manchester, Sackville street building, M60 1QD Manchester, UK
}

Received 28 March 2011 / Received in final form 14 July 2011

Published online 28 September 2011 - (C) EDP Sciences, Società Italiana di Fisica, Springer-Verlag 2011

\begin{abstract}
An optically generated kink observed in the Gummel plot of AlGaAs/GaAs single heterojunction phototransistors (sHPTs) is reported when illuminated with relatively high optical powers. The observed sudden rise in collector current and decrease in the base current, referred to as 'optical kink effect', is carefully studied and analyzed. The measurements are performed for incident optical power of up to $225 \mu \mathrm{W}$ at an incident wavelength of $635 \mathrm{~nm}$. This rise in the current gain of HPTs, in three terminal configuration, is associated with the base-collector space-charge modulation similar to the kirk effect.
\end{abstract}

\section{Introduction}

Heterojunction bipolar phototransistors (HPTs), along with $p$ - $i$ - $n$, avalanche and Schottky photodiodes have been extensively investigated and widely utilized as photodetectors in optical communication and sensor applications [1-3]. The superiority of HPTs over its other counterparts is also well established [4,5]. HPTs exhibit an internal gain, due to transistor action, without high bias voltage as required for avalanche photodiodes and without excess noise due to avalanching [6]. $p-i-n$ and Schottky photodiodes lack internal or intrinsic gain and rely on preamplifiers or subsequent amplification arrangement. HPTs, on the other hand, can provide detection and amplification in a single device along with layer- and process-compatible characteristics with HBT-related technology [7].

Some reports have emerged in recent times which have reported an increased current gain for two terminal (2THPT) operation $[8,9]$. However, in principle three terminal (3T-HPT) operations provide higher gain for phototransistors. In 3T-HPT operation, an additional dc bias at base is used to push the HPT's quiescent bias current to a higher level where the current gain is larger [10]. As HPTs are current controlled devices, the $3 \mathrm{~T}$ optical gain as current-bias HPT configuration is higher than that of a voltage-bias configuration $[11,12]$.

In this work, a nonlinear 'kink' mechanism in the optical gain, at relatively higher incident optical powers, for GaAs-based HPTs is reported. This effect, observed through Gummel plots, occurs due to decrease in the base current and increase in the collector current resulting in a

\footnotetext{
a e-mail: hasan.abbas. khan@gmail . com
}

kink in the optical gain of the transistor. This nonlinearity can have detrimental effect in the opto-microwave matching for three port photodetectors as discussed by Huai et al. [13] and Yo-Sheng and Shey-Shi in [14]. In order to increase the cut off frequency of the photoreceivers, high power or current density operation is desirable which can lead to this non linear effect. This may also limit HPTs functionality in high efficiency high-linearity power amplifiers in various wireless communication systems.

'Electrical kink effect' in HBTs has been widely investigated [15-17]. Liou et al. [15] and Sotodeh et al. [16] associated the observed kink in the base current of single HBTs to forward biasing of base-collector $(\mathrm{B} / \mathrm{C})$ junction. For DHBTs, Tiwari [17] has attributed this effect to the formation of an excess electron barrier in the B/C heterojunction leading to an increase in minority-carrier charge storage in the base and a decrease in current gain of the device. In contrast, the optical kink effect has not been reported for HPTs. This effect, observed in $\mathrm{Al}_{0.3} \mathrm{Ga}_{0.7} \mathrm{As} / \mathrm{GaAs}$ HPTs, is being reported and evaluated here for the first time. We relate this to the Kirk effect, where the carrier concentration due to the high injection of photocarriers from the incident optical power becomes comparable with the native collector doping levels, thus affecting the internal B/C junction voltage.

\section{Device structure and experimental setup}

The $\mathrm{Al}_{0.3} \mathrm{Ga}_{0.7} \mathrm{As} / \mathrm{GaAs}$ Npn HPTs were grown by metalorganic chemical vapor deposition (MOCVD) on GaAs substrate and the devices were fabricated using standard mesa etching and photolithographic techniques. The B/C 
Table 1. Layer structure of the graded base-emitter $\mathrm{Al}_{0.3} \mathrm{Ga}_{0.7} \mathrm{As} / \mathrm{GaAs} \mathrm{Npn}$ transistor.

\begin{tabular}{cccccccc}
\hline Material & $\begin{array}{c}\text { Mole fraction } \\
x(\%)\end{array}$ & $\begin{array}{c}\text { Thickness } \\
(\mu \mathrm{m})\end{array}$ & $\begin{array}{c}\text { Doping } \\
\left(\mathrm{cm}^{-3}\right)\end{array}$ & Type & Dopant & Comments \\
\hline 8 & $\mathrm{GaAs}$ & - & 0.19 & $5 \times 10^{18}$ & $N$ & $\mathrm{Si}$ & Cap layer \\
7 & $\mathrm{Al}_{x} \mathrm{Ga}_{1-x} \mathrm{As}$ & $30-0$ & 0.02 & $5 \times 10^{17}$ & $n$ & $\mathrm{Si}$ & Grading layer \\
6 & $\mathrm{Al}_{x} \mathrm{Ga}_{1-x} \mathrm{As}$ & 30 & 0.15 & $5 \times 10^{17}$ & $n$ & $\mathrm{Si}$ & Emitter \\
5 & $\mathrm{Al}_{x} \mathrm{Ga}_{1-x} \mathrm{As}$ & $0-30$ & 0.02 & $5 \times 10^{17}$ & $n$ & $\mathrm{Si}$ & Grading layer \\
4 & $\mathrm{GaAs}$ & - & 0.09 & $2 \times 10^{19}$ & $p$ & $\mathrm{C}$ & Base \\
3 & $\mathrm{GaAs}$ & - & 0.5 & $2 \times 10^{16}$ & $n$ & $\mathrm{Si}$ & Collector \\
2 & $\mathrm{GaAs}$ & - & 1.0 & $5 \times 10^{18}$ & $n$ & $\mathrm{Si}$ & Sub-collector \\
1 & $\mathrm{GaAs}$ & - & 400 & $\mathrm{U} / \mathrm{D}$ & $S-\mathrm{I}$ & & Substrate \\
\hline
\end{tabular}

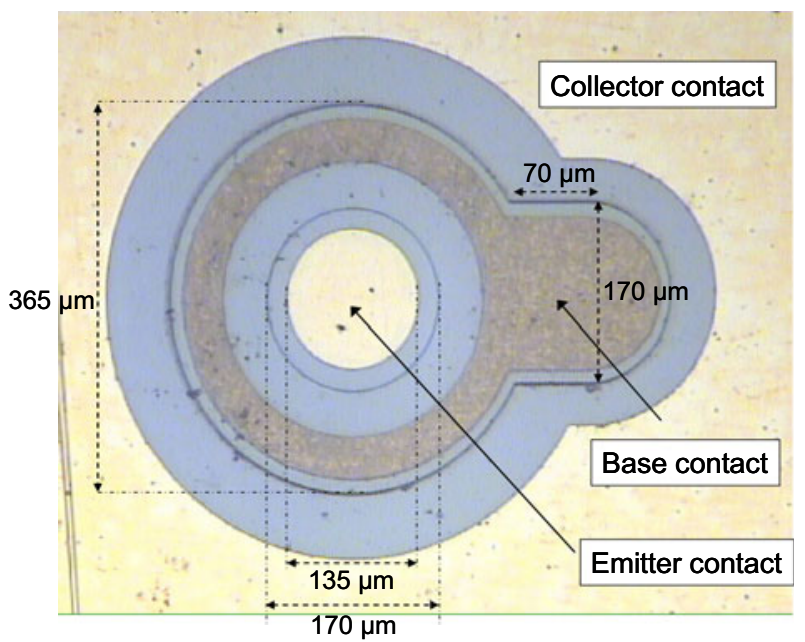

Fig. 1. (Color online) Micrograph of the fabricated $\mathrm{Al}_{0.3} \mathrm{Ga}_{0.7} \mathrm{As} / \mathrm{GaAs} \mathrm{Npn} \mathrm{HPT}$.

and base-emitter $(\mathrm{B} / \mathrm{E})$ junction areas are $2.3 \times 10^{5} \mu \mathrm{m}^{2}$ and $2.3 \times 10^{4} \mu \mathrm{m}^{2}$, respectively. The detailed device layer structure is given in Table 1 .

Layer 1 of the structure is a thick (roughly $400 \mu \mathrm{m}$ ) GaAs substrate which is undoped to keep it semi-insulting. Layer 2, the sub-collector, is highly doped to make low resistance $n$-type $\mathrm{AuGe} / \mathrm{Au}$ ohmic contacts. The collector is lightly doped to keep the breakdown voltage as high as possible during reverse-bias condition. This is followed by a heavily doped GaAs base to provide low base resistance and efficient $p$-type $\mathrm{Au} / \mathrm{Zn} / \mathrm{Au}$ ohmic contacts. The $\mathrm{B} / \mathrm{E}$ junction is graded, hence a couple of thin AlGaAs layers, on either side of the emitter, are present for gradual grading of the junction and to minimize the conduction band discontinuity. The emitter itself is a wide-band gap $\mathrm{Al}_{0.3} \mathrm{Ga}_{0.7} \mathrm{As}$ which is followed by GaAs on the top to facilitate the $n$-type $\mathrm{AuGe} / \mathrm{Au}$ ohmic contact. The micrograph of fabricated device is given in Figure 1 and the absorption area is $1.75 \times 10^{3} \mu \mathrm{m}^{2}$. The detailed experimental set up can be found elsewhere [18].

\section{Results and discussion}

Figure 2 shows the collector and the base currents from the Gummel plots of the HPT illuminated with various opti-

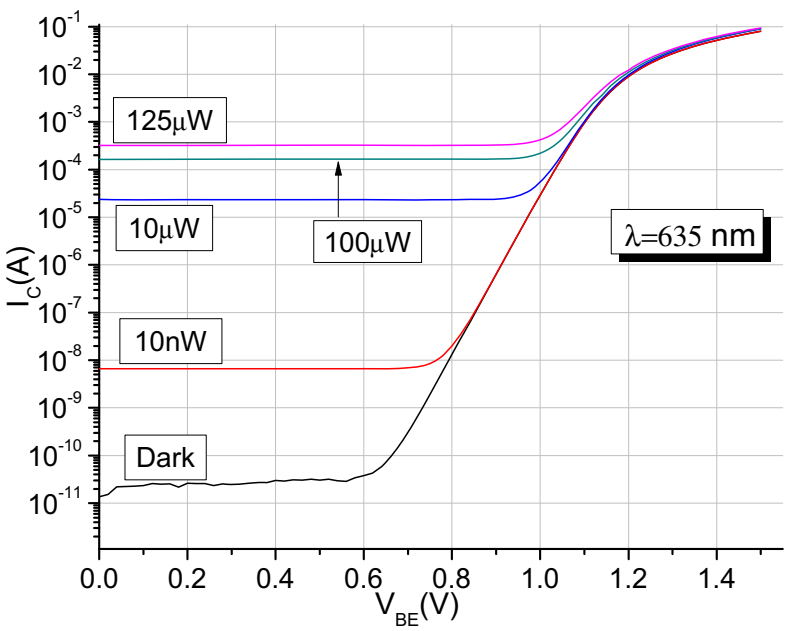

(a)

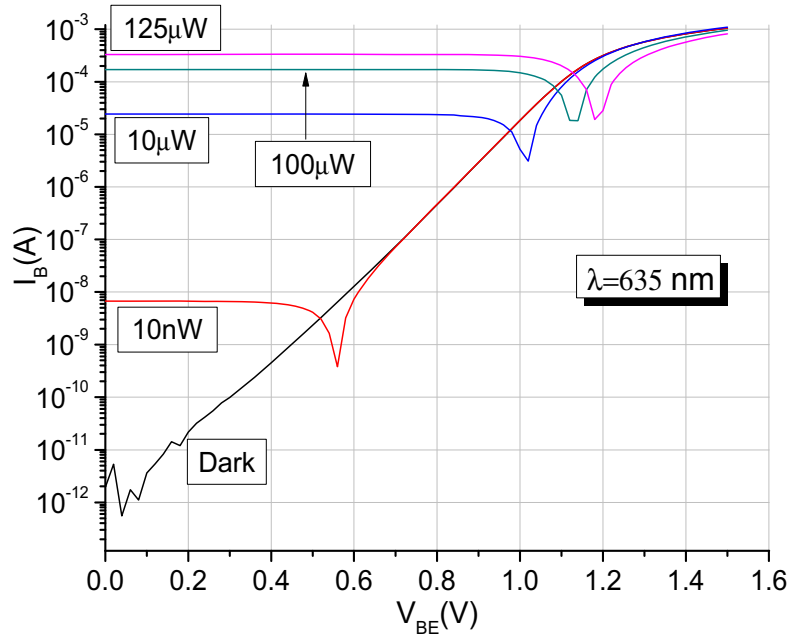

(b)

Fig. 2. (Color online) Collector and Base currents as a function of base-emitter voltage with $V_{B E}$ equal to $V_{B C}$ (Gummel Plot) for $\mathrm{Al}_{0.3} \mathrm{Ga}_{0.7} \mathrm{As} / \mathrm{GaAs}$ Npn HPT at various optical intensities.

cal intensities. Three different regions are presented in this Gummel plot. The first region, at low $V_{B E}$, shows that the dominant current component is the photogenerated one. Both the collector and the base currents saturate at the same level, and the gain in this region is unity. In other words, HPT works as a photodiode. The notch observed 
in the base current corresponds to the point where the external bias current starts to be larger than the photogenerated current and the transistor action begins. The notch shifts towards the right at higher incident optical powers; this is a result of the higher level of photogenerated current, and therefore, it takes more current from the external bias to take over the current due to optical absorption. At this point, diffusion current becomes dominant and the second region starts. In this region, the base supplies holes replacing those lost due to recombination in the base bulk. The third region is when the currents bend to the right, at higher $V_{B E}$, due to presence of the intrinsic series resistances in the phototransistor.

It is also pointed out that the electrical base current is in the reverse direction to the photogenerated current. In other words, the $I_{B}$ at a small $V_{B E}$ shown in Figure $2 \mathrm{~b}$ is a negative value but shown in logarithmic scale. So the measured base current is the difference between the electrical current due to $V_{B E}$ and photogenerated one due to the optical power. Since the electrical current is fixed, the larger optical power results in higher photogenerated current flowing out of the base terminal. Furthermore, at low $V_{B E}$ both the collector and reverse base currents are found to saturate at the value of $I_{p h}$ corresponding to the incident optical power. In addition, we see that the notch in the base current voltage characteristic moves to a higher $V_{B E}$ with increasing optical power. It is noteworthy that these findings are in accordance with Chen et al. [7] and Tan et al. [19] which use analytical models to analyze this effect in the Gummel plots for phototransistors.

In order to analyze various regions of the Gummel plot, Figure 3 has been plotted. Figures $3 \mathrm{a}$ and $3 \mathrm{~b}$ show the behavior of the collector and base currents, respectively at given values of $V_{B E}$ as a function of the optical power applied to the HPT. At $V_{B E}=0.4 \mathrm{~V}$, there is no transistor action taking place and the HPT acts as a $p$ - $i$ - $n$ photodiode. At intermediate $V_{B E}$, the HPT acts as both $p-i-n$ photodiode and a transistor depending on the incident optical power. For instance, at $V_{B E}=1 \mathrm{~V}$, HPT behaves like a transistor at lower optical powers but at high optical powers the photo-absorption swamps the transistor action and the device starts acting as a $p-i$-n photodiode. This detailed analysis on the biasing aspects of HPTs is beneficial for applications in which low power consumption is of prime importance. For instance, optical receivers employing $3 \mathrm{~T}$ phototransistors as the front end detectors can detect lower power for lower values of $V_{B E}$ if the biasing is chosen properly.

At high $V_{B E}(1.5 \mathrm{~V})$, the transistor action dominates, at all optical powers, as the electrical current from the external bias is very large. A slight decrease in base current is observed at high incident optical power which is attributed to 'optical kink effect'. An illustration of this is provided in Figure 4. It can be seen that the base current decreases with increasing optical power. The collector current also increases slightly, at the same time, resulting in the sudden increase in the optical gain of the phototransistor.

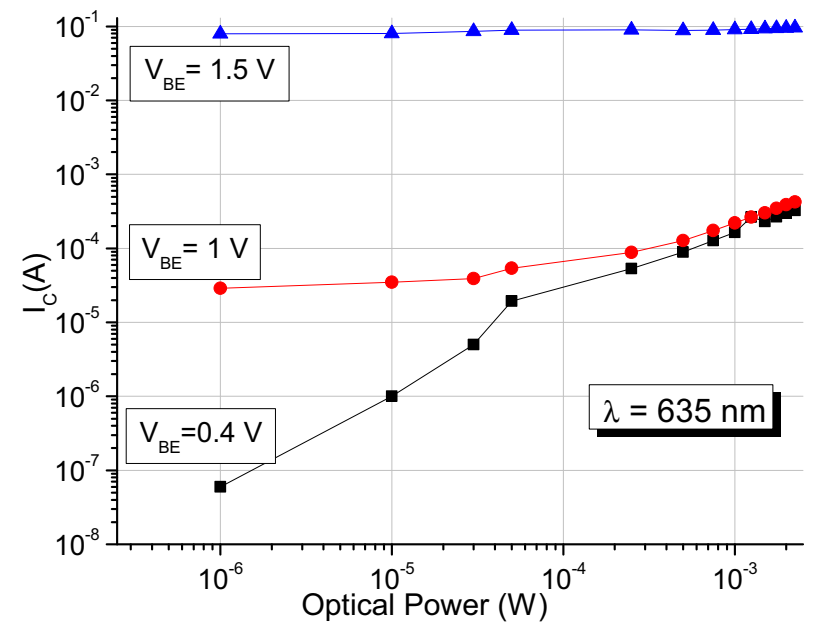

(a)

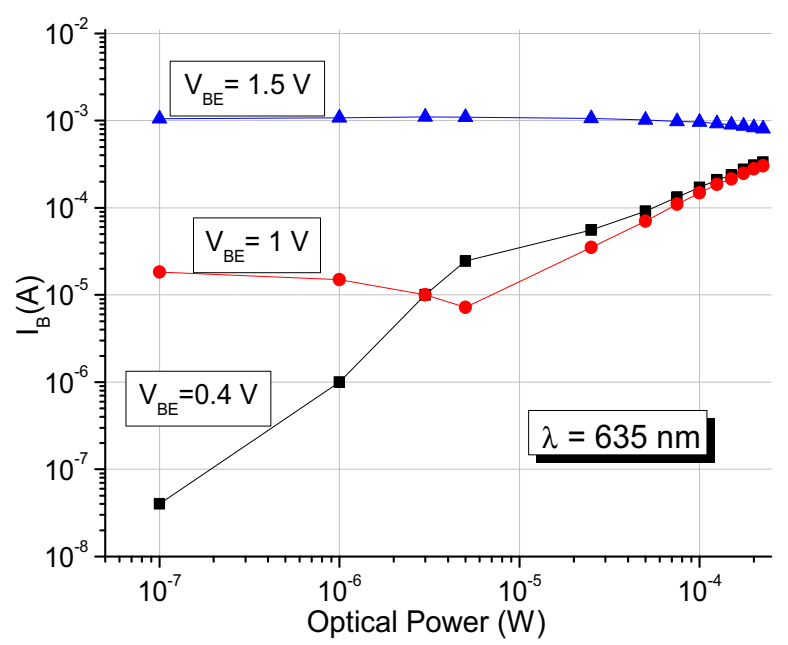

(b)

Fig. 3. (Color online) Measured collector and base currents as a function of optical power at vaious base-emitter voltages for $\mathrm{Al}_{0.3} \mathrm{Ga}_{0.7} \mathrm{As} / \mathrm{GaAs}$ Npn HPT.

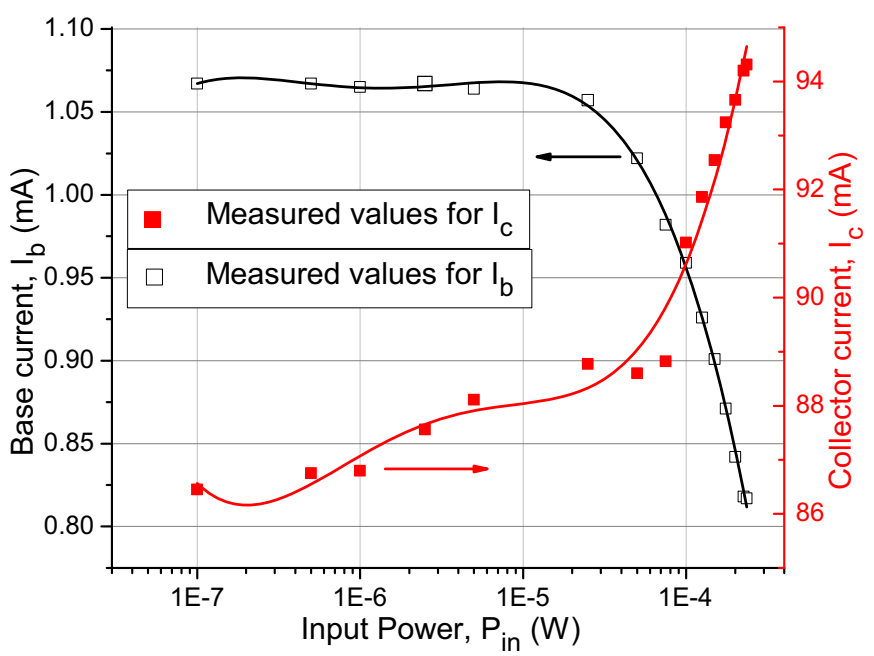

Fig. 4. (Color online) Measured base and collector currents as a function of optical power, for incident wavelength of $635 \mathrm{~nm}$, at $V_{B E}=1.5 \mathrm{~V}$ for $\mathrm{Al}_{0.3} \mathrm{Ga}_{0.7} \mathrm{As} / \mathrm{GaAs} \mathrm{Npn} \mathrm{HPT}$. 
We attribute this nonlinearity to high injection of optical carriers, resulting in an injected photogenerated carrier concentration comparable to the collector native doping concentration; this effect modulates the base-collector depletion region width affecting the currents in the terminals of HPT. The modulation will mostly occur in the collector region as it is lightly doped, with respect to the base region, to limit the breakdown voltage of the $\mathrm{B} / \mathrm{C}$ reverse junction in the forward active mode. In order to investigate this effect further, we calculate the incident flux rate $\left(\phi_{r}\right)$ at the $\mathrm{B} / \mathrm{C}$ junction:

$$
\phi_{r}=\frac{P_{i n}}{E_{p h}} \quad\left[\frac{\text { photons }}{\mathrm{s}}\right]
$$

where $P_{i n}$ is incident optical power and $E_{p h}$ is the energy of the incident photons. For incident power of $100 \mu \mathrm{W}$ at wavelength of $635 \mathrm{~nm}$, the incident photon rate is $1.77 \times$ $10^{14}$ (photons/s). The incident flux per unit volume $\left(\phi_{v}\right)$ in the collector region can be written as:

$$
\phi_{v}=\frac{\phi_{r}}{A W_{C}} \quad\left[\frac{\text { photons }}{\mathrm{s} \mathrm{cm}^{3}}\right]
$$

where $A$ is the absorption area and $W_{C}$, is the collector region width. For $100 \mu \mathrm{W}$ radiation, the $\phi_{v}$ is calculated to be $2.02 \times 10^{23}$ photons $/ \mathrm{s} \mathrm{cm}^{3}$, within the collector region.

This concentration of photons is sufficient enough to neutralize the native doping level in the collector region and therefore the observed optical kink effect is due to the kirk effect in the low doped depleted collector region. In these calculations, Fresnel reflection has been taken into account and the incident powers in Figure 2 is the corrected optical power after incorporation of Fresnel reflection. It should also be noted that many photocarriers recombine before reaching the depleted collection due to surface and interface recombination. In addition, when carrier concentration is very high as a result of either high doping or high injection level, Auger recombination increases, generating current components which add to nonlinearity in the gain of HPTs.

\section{Conclusions}

High injection of optically generated carriers and resulting Kirk effect has been analyzed and related to the cause of optically generated kink effect in $\mathrm{Al}_{0.3} \mathrm{Ga}_{0.7} \mathrm{As} / \mathrm{GaAs} \mathrm{Npn}$ HPTs. Kink effect can result in both non-linear amplification and poor frequency response of phototransistors. This can be minimized by careful optimization of both layer structure and collector doping of HPTs working as frontend detectors for photoreceivers in optical communication.
The authors would like to thank Mr.H. Vladimir Solis for his valuable contribution towards this work. Hassan Khan would also thank Asim Khan, K. Karimov and Tauseef Tauqeer and for their valuable input towards this research.

\section{References}

1. S.W. Choi, S. Furue, N. Hayama, K. Nishida, M. Ogura, IEEE Photon. Technol. Lett. 21, 1187 (2009)

2. M.A. Cappelletti, A.P. Cedola, E.L. Peltzer, Y. Blanca, Semicond. Sci. Technol. 23 (2008)

3. C. Gonzales, Presented at the International symposium on microwave photonics (invided paper) (Budapest, Hungary, 2003)

4. T.F. Refaat, M.N. Abedin, O.V. Sulima, S. Ismail, U.N. Singh, Presented at the Infrared Detectors and Focal Plane Arrays VIII (San Diego, CA, USA, 2006)

5. V.M.S. Dupont, E. Fendler, F. Jorge, S. Maricot, J.-P. Vilcot, J. Harari, D. Decoster, Presented at the Diamond Congress (Invited paper) (Budapest, Hungary, 2004)

6. J. Van de Casteele, V. Magnin, J.P. Gouy, J.P. Vilcot, J. Harari, S. Maricot, D. Decoster, Presented at the Photodetectors: Materials and Devices II (USA, 1997)

7. H.R. Chen, W.T. Chen, M.K. Hsu, S.W. Tan, W.S. Lour, Semicond. Sci. Technol. 20, 548 (2005)

8. P. Min Su, J. Jae Hyung, IEEE Photon. Technol. Lett. 22, $1202(2010)$

9. Y.W. Byun, M.S. Park, Y.H. Park, Y.C. Cho, J.H. Jang, Presented at the IEEE International Conference on Indium Phosphide and Related Materials, IPRM 2009 (Newport Beach, CA, USA, 2009)

10. S.W. Tan, H.R. Chen, A.H. Lin, W.T. Chen, W.S. Lour, Semicond. Sci. Technol. 19, 1213 (2004)

11. H.R. Chen, S.W. Tan, W.T. Chen, T.S. Lin, W.S. Lour, Presented at the 2004 Conference on Optoelectronic and Microelectronic Materials and Devices. Proceedings (Piscataway, NJ, USA, 2004)

12. H.R. Chen, S.W. Tan, A.H. Lin, W.T. Chen, W.S. Lour, presented at the IVESC2004 - 5th International Vacuum Electron Sources Conference Proceedings (Beijing, China, 2004)

13. G. Huai, S. Xiaohong, H. Yunan, Z. Xiaodong, W. Rong, G.P. Li, IEEE Electron Device Lett. 31, 1113 (2010)

14. L. Yo-Sheng, L. Shey-Shi, IEEE Trans. Electron Devices $\mathbf{5 2}, 132(2005)$

15. J.J. Liou, L.L. Liou, Solid-State Electron. 36, 1222 (1993)

16. M. Sotoodeh, A.H. Khalid, A.A. Rezazadeh, Solid-State Electron. 42, 531 (1998)

17. S. Tiwari, IEEE Electron Device Lett. 9, 142 (1988)

18. H.A. Khan, A.A. Rezazadeh, S. Sohaib, J. Appl. Phys. 109, 104507 (2011)

19. S.W. Tan, H.R. Chen, W.T. Chen, M.K. Hsu, A.H. Lin, W.S. Lour, J. Appl. Phys. 97, 034502 (2005) 